\title{
Microarray analysis of the transcriptional responses of Clostridium difficile to environmental and antibiotic stress
}

\author{
Jenny E. Emerson, ${ }^{1}$ Richard A. Stabler, ${ }^{2}$ Brendan W. Wren ${ }^{2}$ \\ and Neil F. Fairweather ${ }^{1}$ \\ ${ }^{1}$ Centre for Molecular Microbiology and Infection, Division of Cell and Molecular Biology, Imperial \\ College London, London SW7 2AZ, UK \\ ${ }^{2}$ Department of Infectious and Tropical Diseases, London School of Hygiene and Tropical Medicine, \\ Keppel Street, London WC1E 7HT, UK
}

Neil F. Fairweather

n.fairweather@imperial.ac.uk

\begin{abstract}
Clostridium difficile is a spore-forming anaerobic bacterium that is an emerging nosocomial threat; incidence of infection in hospitals is increasing, both in frequency and severity, resulting in considerable morbidity and mortality. In order to adapt to the intestinal environment, C. difficile must react to the many stresses involved with colonization, including exposure to antibiotics, which represents the most frequent precipitating agent of $C$. difficile infection. The responses of C. difficile to environmental shocks (heat, $\mathrm{pH}$ and oxidative shock) and to growth in the presence of subinhibitory concentrations of antibiotics (amoxicillin, clindamycin and metronidazole) were investigated using the C. difficile 630 microarray developed by the Bacterial Microarray Group at St George's, University of London, UK (B $\mathrm{G} @ @ S)$. Significantly regulated genes and operons were identified that are unique to or common between different stresses. The transcriptional profiles of C. difficile 630 are similar after growth in the presence of amoxicillin and clindamycin: both increased transcription of ribosomal protein genes and altered transcription of genes encoding surface-associated proteins. In contrast, metronidazole treatment resulted in minor changes in transcription patterns. The general stress response is observed after heat shock and acid shock. Heat shock also affected transcription of several biochemical pathways. Exposure to atmospheric oxygen induced a large number of electron transporters. This study provides a starting point for detailed analyses of numerous genes whose expression is affected by stress and may therefore be involved in adaptation to the host environment.
\end{abstract}

Received 27 September 2007

Accepted 25 January 2008

\section{INTRODUCTION}

Clostridium difficile is a spore-forming anaerobic bacterium that primarily causes antibiotic-associated diarrhoea, but disease may be precipitated with any agent that disrupts the normal colonic microflora (Bartlett, 2006). Incidence in hospitals is increasing, both in frequency and severity, resulting in considerable morbidity and mortality. Recent outbreaks in Canada, the USA and the UK have highlighted the severity of this disease (Loo et al., 2005; McDonald et al., 2005; Warny et al., 2005). Although C. difficile is

\footnotetext{
Abbreviations: CDAD, Clostridium difficile-associated disease; CWP, cell wall protein.

Fully annotated microarray data are available in B $\mu \mathrm{G} @$ Sbase (http://bugs. sgul.ac.uk) and also ArrayExpress (http://www.ebi.ac.uk/arrayexpress/) with accession numbers E-BUGS-55 (environmental shock) and E-BUGS56 (growth in the presence of antibiotics).
}

Tables of gene expression data are available as supplementary data with the online version of this paper. resistant to many of the antibiotics that are strongly linked to infection, particularly in the case of quinolones and the emergent ribotype 027 strains, resistance to the precipitating agent is not necessary for $C$. difficile-associated disease (CDAD) to occur (Freeman \& Wilcox, 2001). In order to more fully understand the changes to the bacterium induced by antibiotics, we have used microarray technology to analyse transcriptional responses of $C$. difficile 630 to growth in the presence of subinhibitory concentrations of antibiotics. Three antibiotics were chosen for this study: amoxicillin, a commonly prescribed drug that can precipitate $\mathrm{CDAD}$; clindamycin, the original antibiotic linked to CDAD; and metronidazole, often the first line of treatment for CDAD.

To understand how $C$. difficile interacts with its host, it is necessary to elucidate the survival mechanisms necessary for colonization and pathogenesis, and for persistence outside the host. During infection of the host C. difficile is exposed to the low $\mathrm{pH}$ environment of the stomach and 
high $\mathrm{pH}$ bile salts, in addition to various oxidative stresses. Analysis of the responses of $C$. difficile to general stresses will prove useful in revealing possible adaptation and resistance mechanisms in vivo.

The general mechanism of the heat-shock response is conserved in all organisms (Boorstein et al., 1994; Gupta, 1995), and in Gram-positive bacteria it consists of three major regulons: HrcA, CtsR and SigB (reviewed by Rosen \& Ron, 2002; Schumann, 2003). These responses are not solely regulated by heat shock, and may be induced by any stress producing damaged or denatured proteins, for example ethanol, acid or salt shocks. This can be seen in Clostridium acetobutylicum, where butanol stress induces several heat-shock genes (Tomas et al., 2004). The heatshock-induced sigma $\mathrm{B}\left(\mathrm{SigB}, \sigma^{\mathrm{B}}\right)$ regulon varies between different organisms, and can incorporate as many as 200 genes (Hoper et al., 2005; Pane-Farre et al., 2006; van Schaik et al., 2007). Some genes (e.g. $c l p P$ and $c l p C$ ) are regulated by multiple mechanisms (Petersohn et al., 2001).

Previous studies in $C$. difficile of the heat-shock protein GroEL have shown both the mRNA and protein to be strongly induced after heat shock from 30 to $43{ }^{\circ} \mathrm{C}$; and GroEL was also secreted into the supernatant. However, no change in expression is observed after heat shock from 30 to $48{ }^{\circ} \mathrm{C}$ (Hennequin et al., 2001). Acidic conditions and high osmolarity also strongly induced groEL mRNA but this was not accompanied by a large relative increase in protein levels (Hennequin et al., 2001). In this study, we have used microarray analysis to determine the proportional changes in the entire transcriptome of $C$. difficile 630 in response to heat, oxidative, acid or alkaline shocks in addition to growth in the presence of antibiotics.

\section{METHODS}

Bacterial strains and culture conditions. C. difficile 630 (Wust \& Hardegger, 1983) was provided by Dr Peter Mullany, Eastman Dental Institute, London, UK, and has been fully sequenced by the Wellcome Trust Sanger Institute (Sebaihia et al., 2006). C. difficile was routinely cultured either on blood agar base supplemented with $7 \%$ horse blood or in brain heart infusion (BHI) broth. Culture was undertaken in an anaerobic cabinet at $37{ }^{\circ} \mathrm{C}$ (or $30{ }^{\circ} \mathrm{C}$ for heat-shock experiments) in a reducing anaerobic atmosphere $\left(10 \% \mathrm{CO}_{2}, 10 \%\right.$ $\mathrm{H}_{2}, 80 \% \mathrm{~N}_{2}$ ). To obtain exponential phase cells, an overnight liquid culture was inoculated $1 / 20$ into BHI that had been pre-equilibrated to the desired temperature and anaerobic atmosphere, and shaken to mix thoroughly. Growth of $C$. difficile was monitored by measurement of $\mathrm{OD}_{600}$ and the cultures were treated between $\mathrm{OD}_{600} 0.5$ and 0.7 .

Stress induction. All experiments were carried out in biological triplicate. For growth in the presence of antibiotics, an overnight liquid culture was inoculated into BHI containing either $1 \mu \mathrm{g}$ amoxicillin $\mathrm{ml}^{-1}, 50 \mu \mathrm{g}$ clindamycin $\mathrm{ml}^{-1}$ or $0.15 \mu \mathrm{g}$ metronidazole $\mathrm{ml}^{-1}$. The cells were harvested between $\mathrm{OD}_{600} 0.5$ and 0.7 .

For heat-shock experiments, cultures were grown in BHI broth at $30{ }^{\circ} \mathrm{C}$ to $\mathrm{OD}_{600} 0.5-0.7$. Aliquots $(15 \mathrm{ml})$ were sealed into $50 \mathrm{ml}$ Falcon tubes and incubated in a water bath at either $30{ }^{\circ} \mathrm{C}$ (control) or $42{ }^{\circ} \mathrm{C}$ (heat) for 15 min before harvest.
For $\mathrm{pH}$-shock experiments, it was determined that $C$. difficile could withstand $\mathrm{pH}$ shock between $\mathrm{pH} 4.8$ and $\mathrm{pH}$ 8.3. Aliquots $(15 \mathrm{ml})$ of exponential phase culture were added to $50 \mathrm{ml}$ Falcon tubes containing $0.5 \mathrm{ml} \mathrm{BHI}$ with either $63 \mu \mathrm{l} 10 \mathrm{M}$ sodium hydroxide (designed to alter the final $\mathrm{pH}$ to $\mathrm{pH} 8.5$ ), $24 \mu \mathrm{l}$ concentrated hydrochloric acid plus $39 \mu \mathrm{l}$ water (to $\mathrm{pH} 4.5$ ), or $63 \mu \mathrm{l}$ water, and then incubated anaerobically for $15 \mathrm{~min}$ before harvest.

For oxygen exposure experiments, $15 \mathrm{ml}$ aliquots were sealed into $50 \mathrm{ml}$ Falcon tubes and removed from the anaerobic cabinet. Half of the aliquots were exposed to the atmosphere and then resealed and shaken, three times. Control aliquots remained sealed with Parafilm and were shaken but not exposed to the atmosphere. All aliquots were then Parafilm sealed and returned to the $37^{\circ} \mathrm{C}$ anaerobic cabinet for $10 \mathrm{~min}$ - it was determined that at least $15 \%$ of cells survived this treatment.

After treatment, in each case two volumes of RNAprotect bacteria reagent (Qiagen) were immediately added to cells, mixed, and incubated for $5 \mathrm{~min}$. Cells were harvested by centrifugation and the cell pellet was stored at $-80{ }^{\circ} \mathrm{C}$.

RNA extraction and real-time PCR. RNA was extracted using a TRIzol Max bacterial RNA isolation kit (Invitrogen) according to the manufacturer's instructions, and then DNase treated. A 16S rRNA PCR amplification was carried out using $0.5 \mu \mathrm{l}$ RNA as template to ensure there was no residual DNA.

For real-time PCR, RNA was reverse transcribed using a high-capacity cDNA archive kit (Applied Biosystems) according to the manufacturer's instructions. Custom TaqMan gene expression assays were designed by Applied Biosystems and carried out according to the manufacturer's instructions.

Microarray analysis. In 2004, the Bacterial Microarray Group at St George’s, University of London, London, UK (B $\mu \mathrm{G} @ S)$ designed a microarray based on the preliminary C. difficile 630 genome sequence and annotation available at the time. The microarray is described by Stabler et al. (2006). The B $\mu \mathrm{G} @ S$ CDv1.0.0 array design is available in B $\mu \mathrm{G} @ S b a s e$ (accession number A-BUGS-20; http://bugs.sgul.ac.uk/ABUGS-20) and also ArrayExpress (accession number A-BUGS-20). To aid in interpretation of the results, the predicted proteome was subjected to large batch Pfam analysis (http://pfam.sanger.ac.uk); the detected domains are presented in Supplementary Tables S1-S14 (available with the online journal).

RNA was concentrated using an RNeasy MinElute cleanup kit (Qiagen) according to the manufacturer's instructions and then adjusted to $1.25 \mu \mathrm{g}^{-1}$. Duplicate competitive genomic DNA/ RNA microarrays were then carried out according to the standard B $\mu \mathrm{G} @ S$ hybridization protocol. The slides were scanned using the standard B $\mu \mathrm{G} @ S$ Affymetrix 428 scanning protocol, and fluorescence was quantified and flagged using ImaGene v5.5 (BioDiscovery). For antibiotic stress data, datasets from images at multiple intensities were combined using MAVI Pro v2.6.0 (MWG Biotech) to create a single dataset with larger dynamic range than is possible to determine from a single image.

Paired datasets for Cy5 (RNA) and Cy3 (DNA) microarray element fluorescence intensity quantifications were then uploaded to GeneSpring GX v6.1 (Agilent Technologies). Values below 0.01 were set to 0.01 , to allow logarithmic interpretation of the data. The $\log _{2}$ ratio of the intensity in the RNA channel (Cy5) to that in the control DNA channel (Cy3) was calculated. Data were then normalized to each slide's median intensity ratio (of present and marginal microarray elements). Each gene in each experiment was then normalized to the mean of the ratios obtained under its 'control' condition. The default interpretation mode was set to 'log of ratio' (of present and marginal microarray elements) to allow correct parametric statistical analysis. Each stress was 
then tested separately for statistical differences between stress and control conditions using ANOVA. A 1 or $5 \%$ confidence level was used, in conjunction with Benjamini-Hochberg, Bonferroni or no multiple testing correction.

Fully annotated microarray data for environmental-shock response has been deposited in B $\mu$ G@Sbase (http://bugs.sgul.ac.uk/E-BUGS-55) and also in ArrayExpress (http://www.ebi.ac.uk/arrayexpress/) with accession number E-BUGS-55; and for growth in the presence of antibiotics with accession number E-BUGS-56.

\section{RESULTS AND DISCUSSION}

Cultures of C. difficile 630 were grown to exponential phase in the presence of subinhibitory concentrations of antibiotics. Alternatively, exponential phase cultures were subjected to $15 \mathrm{~min}$ of heat shock (from 30 to $42{ }^{\circ} \mathrm{C}$ ), 15 min of $\mathrm{pH}$ shock (by $\sim 1.5 \mathrm{pH}$ units), or 10 min of atmospheric exposure. RNA was extracted and analysed by competitive RNA/DNA microarray using the B $\mu \mathrm{G} @ \mathrm{~S}$. difficile 630 microarray. ANOVA statistical analysis of the behaviour of each microarray spot reveals that distinct (but overlapping) gene sets are significantly regulated after each stress, as shown in Table 1. This paper describes a selection of the results obtained from the study, concentrating on several groups of genes whose expression is altered by environmental shock or antibiotic treatment. Complete lists of statistically regulated genes are given in Supplementary Tables S1-S14 (available with the online journal). A summary of the behaviour of every gene after every stress, including each gene's annotation data and our Pfam domain analysis, are given in Supplementary Tables S15-S16 (available with the online journal).

As expected, heat shock of $C$. difficile results in upregulation of gene clusters homologous to known E. coli heat-shock operons; all are significant using the more stringent Bonferroni multiple testing correction. This verifies both the environmental stress procedures and the microarray data validity. The HrcA-regulated gene cluster containing dnaJ,
dnaK, grpE, hrcA and hemN is co-ordinately upregulated in response to heat. The CtsR-regulated $c l p C$ is situated in a gene cluster with $c s t R$ itself and genes encoding a DNA repair protein and an ATP : guanidophosphotransferase; this cluster appears to be co-ordinately upregulated, as are groES and groEL (Fig. 1a). $\operatorname{clpB}$ is located in a cluster with genes encoding two hypothetical proteins, which are also significantly upregulated. $h t p G$, encoding a heat-shock chaperone protein, is upregulated by heat shock, and $\operatorname{csp} D$, encoding a cold-shock protein, is downregulated. Analysis by real-time PCR of groEL expression over a time-course of heat shock reveals that it is induced most strongly at $15 \mathrm{~min}$ (13-fold, $P<10^{-5}$ ), and is also significantly upregulated at all other time points examined (5, 30 and $60 \mathrm{~min} ;>3$-fold induction, $P<10^{-3}$ ) (Fig. 1b). The heat-shock gene clusters are also upregulated after acid shock (Fig. 1a). The characterization of the response of the $C$. difficile vegetative cell to acid shock may have direct relevance to CDAD precipitated by gastric acid suppressants; in these patients gastric $\mathrm{pH}$ can rise above pH 5 (Jump et al., 2007), and we have shown vegetative $C$. difficile to be able to tolerate this.

Heat shock would also be expected to upregulate the sigB$r s b V-r s b W$ operon, but this was not observed in these microarray hybridizations. This may be explained by the time point examined: $\operatorname{sig} B$ in Bacillus subtilis is observed to be maximally induced $3 \mathrm{~min}$ after the onset of heat shock, and the degree of upregulation is greatly reduced after 10 and 20 min of heat shock (Helmann et al., 2001).

Genes encoding four enzymes of the spermidine synthesis pathway - speA (encoding arginine decarboxylase), speD (encoding $S$-adenosylmethionine decarboxylase proenzyme), speE (encoding spermidine synthase), and speB (encoding putative agmatinase) - are situated in a cluster within the genome. All four genes are upregulated on average 3.1-fold in response to heat shock (Fig. 2a). Additionally, genes encoding the four components of the spermidine/putrescine $\mathrm{ABC}$ transporter - potA (encoding

Table 1. Numbers of genes significantly regulated by shock

The total number of genes determined to be upregulated or downregulated by each stress was determined using microarray analysis.

\begin{tabular}{|lclrr|}
\hline Stress & Significance (\%) & Multiple testing correction & Upregulated & Downregulated \\
\hline Heat & 5 & Benjamini-Hochberg & 114 & 98 \\
& 5 & Bonferroni & 64 & 31 \\
Acid & 1 & None & 24 & 5 \\
& 5 & Benjamini-Hochberg & 4 & 0 \\
Alkali & 1 & None & 33 & 10 \\
Oxygen & 1 & None & 98 & 26 \\
& 5 & Benjamini-Hochberg & 19 & 0 \\
Amoxicillin & 1 & Benjamini-Hochberg & 230 & 24 \\
Clindamycin & 5 & Bonferroni & 11 & 1 \\
Metronidazole & 1 & None & 74 & 69 \\
& 1 & None & 8 & 12 \\
\hline
\end{tabular}


(a)

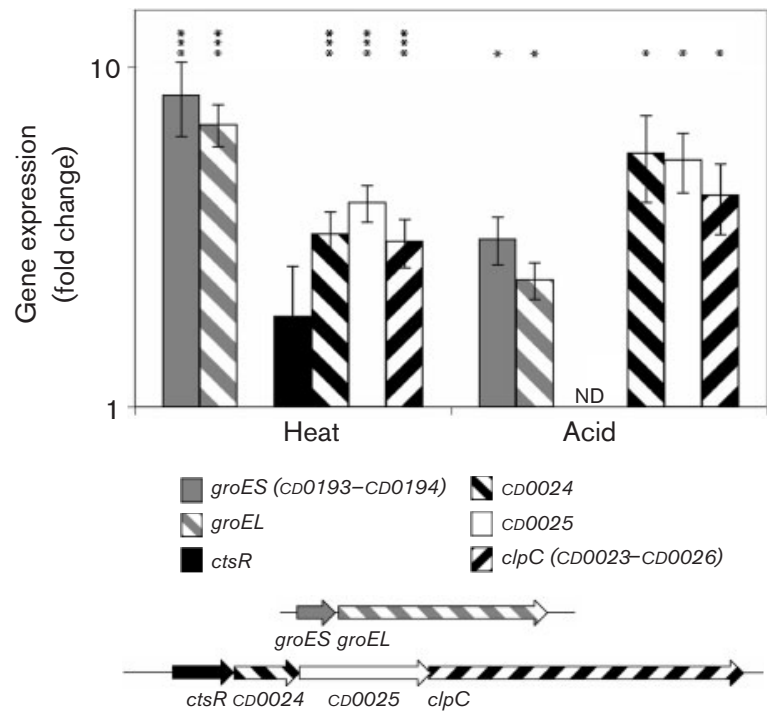

(b)

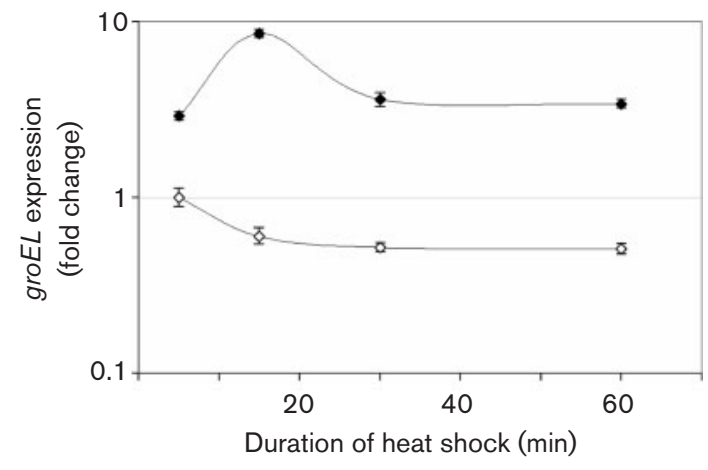

Fig. 1. Regulation of heat-shock protein genes by environmental shock. (a) Microarray gene expression data represented as fold change with respect to control conditions. SE is shown. *, Significant using ANOVA with $1 \%$ significance; ${ }^{* * *}$, significant using ANOVA with $5 \%$ significance and Bonferroni multiple testing correction. The operon structure of these gene clusters within the genome is shown below the graph. ND, No data. (b) Real-time PCR analysis of the expression of groEL over a time-course of heat shock. Data were normalized to $\operatorname{s} p A$ expression and then converted to fold change from the expression after $5 \mathrm{~min}$ incubation at $30{ }^{\circ} \mathrm{C}$. Error bars represent SE of triplicate measurements of triplicate biological samples. $42{ }^{\circ} \mathrm{C} ; \diamond, 30{ }^{\circ} \mathrm{C}$.

ATP-binding protein), potB (encoding permease protein), potC (encoding putative permease protein) and potD (encoding substrate-binding lipoprotein) - are located in a cluster with a gene encoding a putative transcriptional regulator, and all five genes are co-ordinately upregulated by heat shock by a mean of 3.3-fold (Fig. 2a). These spermidine synthesis and spermidine/putrescine $A B C$ transporter gene clusters are also co-ordinately upregulated in response to alkali shock by a mean of 2.5- and 3.0-fold, respectively. Analysis by real-time PCR of the expression of speE (Fig. 2b) and potC (data not shown) over a

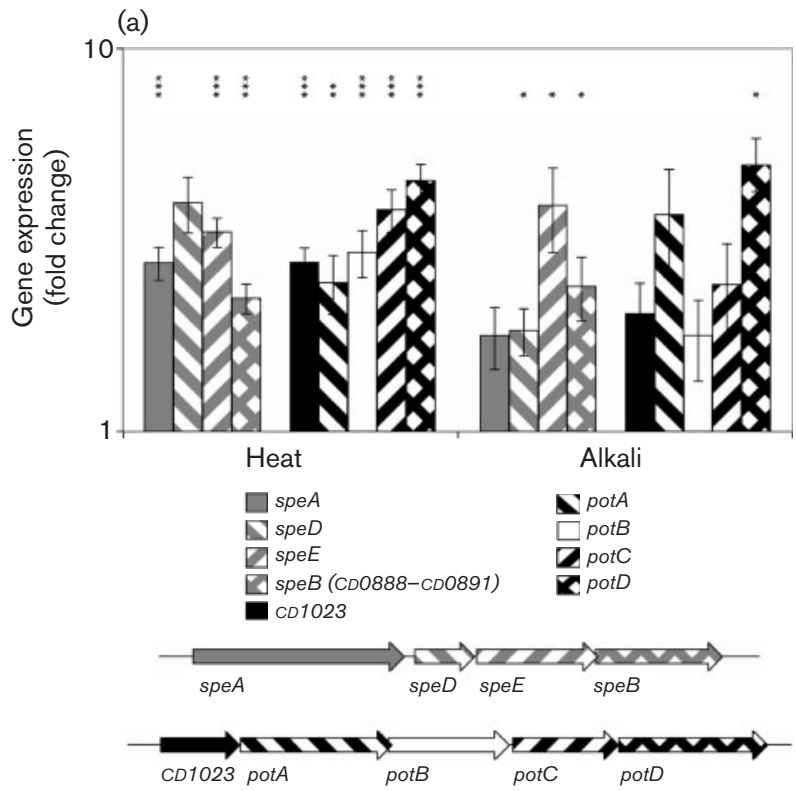

(b)

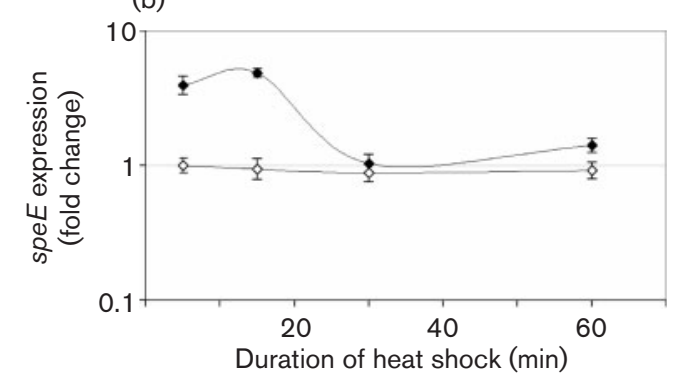

Fig. 2. Regulation of spermidine-related genes by environmental shock. (a) Microarray gene expression data represented as fold change with respect to control conditions. SE is shown. *, Significant using ANOVA with $1 \%$ significance; ${ }^{* *}$, significant using ANOVA with $5 \%$ significance and Benjamini-Hochberg multiple testing correction; ${ }^{* * *}$, significant using ANOVA with $5 \%$ significance and Bonferroni multiple testing correction. The operon structure of the gene clusters within the genome is shown below the graph. (b) Real-time PCR analysis of the expression of $s p e E$ over a time-course of heat shock. Data were normalized to $s / p A$ expression and then converted to fold change from the expression after $5 \mathrm{~min}$ incubation at $30^{\circ} \mathrm{C}$. Error bars represent SE of triplicate measurements of triplicate biological samples. $\$ 42^{\circ} \mathrm{C}$; $\diamond, 30^{\circ} \mathrm{C}$.

time-course of heat shock reveals that significant induction is observed after 5 and 15 min: speE 4.1-fold $\left(P<10^{-3}\right)$ and 5.0-fold $\left(P<10^{-4}\right)$; and potC 7.2-fold $\left(P<10^{-5}\right)$ and 5.6fold $\left(P<10^{-3}\right)$, respectively. No significant induction of either gene is seen at 30 or $60 \mathrm{~min}$ of heat shock. Spermidine is a polyamine involved in various cellular processes, and is linked to abiotic stress resistance in apple and pear fruit trees (Wen et al., 2007), and peroxide resistance in mouse fibroblasts (Rider et al., 2007). The Pot ABC transporter is known to be surface associated in Streptococcus pneumoniae 
(Shah et al., 2006) and may contribute to virulence in a murine sepsis model (Ware et al., 2006).

In B. subtilis, the alkali-shock response is mediated by the RNA polymerase alternative sigma factor sigma W (SigW, $\sigma^{\mathrm{W}}$ ) (Wiegert et al., 2001). However, BLASTP searching of the C. difficile 630 and R20352 (partial) published genome sequences reveals that there is no close homologue of $\sigma^{\mathrm{W}}$ in C. difficile. Similarities between the alkali responses of $C$. difficile and $B$. subtilis can still be noted. The histidine synthesis pathway has previously been detected as being upregulated after alkaline shock (Wiegert et al., 2001), and this was also observed in this study. Tellurite and tellurium resistance proteins are upregulated in B. subtilis (Petersohn et al., 2001; Wiegert et al., 2001), and these genes were upregulated by a mean of 1.4 -fold by alkali shock in this study, although none significantly so. However, induction of $\mathrm{Na}^{+} / \mathrm{H}^{+}$antiporters and multidrug efflux protein encoding genes was not observed, and neither was any evidence of the phosphate starvation observed in Bacillus licheniformis by Hornbaek et al. (2004).

Despite being regarded as strictly anaerobic, C. difficile does appear to respond specifically to the oxidative stress of atmospheric exposure. In total, $7.6 \%(18 / 238)$ of genes annotated as encoding proteins involved in electron transport are significantly upregulated. These include a gene cluster encoding a putative oxidoreductase (NAD/ FAD binding subunit, electron transfer subunit and acetylCoA synthase subunit) that is co-ordinately upregulated (Fig. 3). Genes encoding a putative nitric oxide reductase flavoprotein and NorV (a putative anaerobic nitric oxide reductase flavorubredoxin), which may be involved in protection against nitric oxide, are also upregulated (Fig. 3 ), as are other redox proteins including thioredoxins, thiol peroxidase and rubrerythrin.

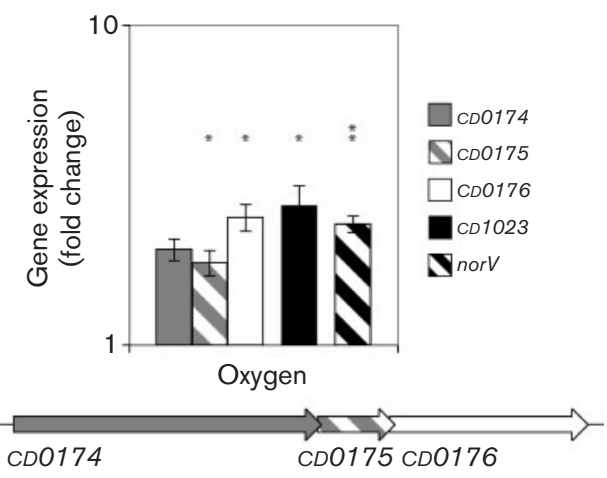

Fig. 3. Regulation of oxidoreductase genes by environmental shock. Microarray gene expression data represented as fold change with respect to control conditions. SE is shown. *, Significant using ANOVA with $1 \%$ significance; ${ }^{\star *}$, significant using ANOVA with $5 \%$ significance and Benjamini-Hochberg multiple testing correction. The operon structure of the gene clusters within the genome is shown below the graph - the nitric oxide reductase flavoproteins do not lie in a cluster in the genome.
In addition to the upregulation of redox proteins, much of the response of $C$. difficile 630 to atmospheric exposure is the regulation of genes encoding proteins of as yet unknown function: of the 341 conserved hypothetical protein-encoding genes in the genome 18 are significantly upregulated, as are 7 of the 164 hypothetical protein-encoding genes represented on the microarray. This highlights the fact that many of the response mechanisms of $C$. difficile to oxygen exposure are highly uncharacterized. The conserved nature of these proteins implies that these responses to oxidizing conditions may also be found in other organisms, and could represent shared survival mechanisms.

The observed response of $C$. difficile to atmospheric exposure is very interesting. The induction of a large number of presumably protective oxidoreductases and electron transport components implies that $C$. difficile does in fact have some mechanisms for tolerance of limited oxidizing conditions. The low viability of our atmospherically exposed cultures suggests that $C$. difficile 630 is substantially damaged by the level of oxygen exposure used in this study. However, the observed responses may promote survival, if not growth, in microaerophilic environments. The gut is not strictly anaerobic, and C. difficile either must grow and survive in strictly anaerobic niches or must be capable of tolerating microaerophilic conditions. It is interesting to note that sporulation does not appear to be induced by the environmental stresses tested, but this may be explained by the time point tested - no observed sporulation would be expected after 15 min of stress.

Growth in the presence of all three antibiotics tested resulted in the general upregulation of transcription and translation machinery. The genome region CD0059-CD0099 contains genes encoding many ribosomal proteins and other growth-related proteins. This region appears to be co-ordinately upregulated by amoxicillin (with the exception of $C D 0065$, which is unchanged) by a mean of 4.4 -fold (range 2.2- to 6.0-fold) (Fig. 4a). This includes significant upregulation of rpoB, rpoC and rpoA (encoding RNA polymerase $\beta, \beta^{\prime}$ and $\alpha$ chains) and 25 ribosomal proteinencoding genes (plus 4 situated elsewhere). Overall, $30 \mathrm{~S}$ and $50 \mathrm{~S}$ ribosomal protein genes are induced by $3.7-$ and 3.8 -fold, respectively, after growth in the presence of amoxicillin. Five tRNA synthetases are significantly upregulated after amoxicillin treatment, and genes encoding tRNA-associated proteins are on average upregulated 1.8fold. The CD0059-CD0099 region is also co-ordinately upregulated after both clindamycin and metronidazole exposure, although no genes significantly so. Taken together these results imply that a general increase in transcription and translation may be a response to the presence of antibiotics, particularly amoxicillin.

Transcriptional upregulation of most genes encoding ribosomal proteins, and also the majority of tRNA synthetases, has also been observed after antibiotic shock of $B$. subtilis using protein synthesis inhibitors (Lin et al., 2005). Some antibiotics that inhibit transcription and 
(a)

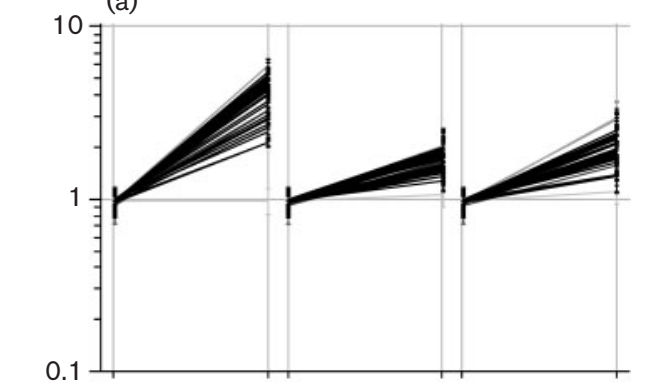

(b)

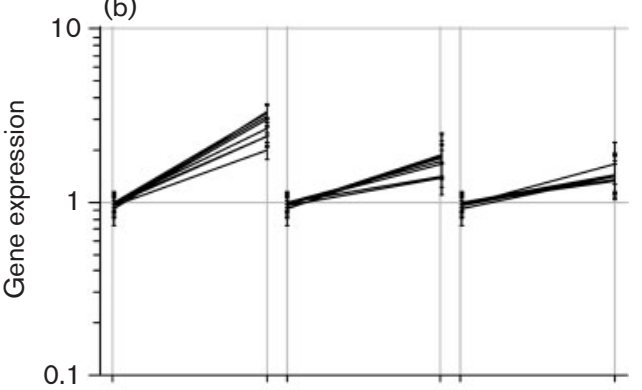

(c)

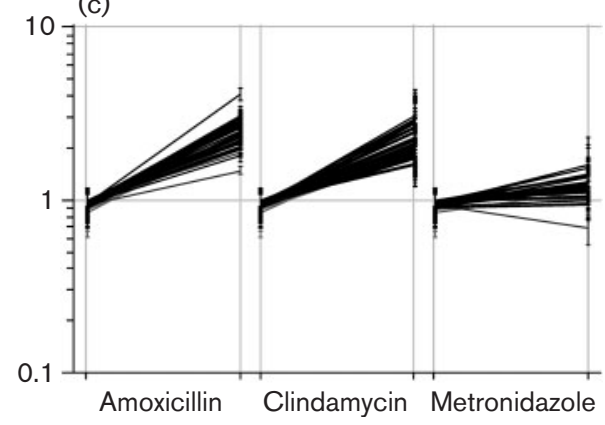

Fig. 4. Responses of ribosomal protein, CWP- and flagellarencoding genes to growth in the presence of antibiotics. Gene expression levels were determined by microarray analysis and normalized to 1 under control conditions (no antibiotic is shown at the left of each panel). Fold-change after growth in the presence of antibiotics is represented to the right of each panel. Each line represents data for one microarray reporter, with SE bars, and the darkness of the line represents reliability of the data. (a) Each line represents one gene within the CD0059-CD0099 genome region, encoding primarily ribosomal proteins. (b) Each line represents one microarray reporter: $s / p A, c w p 2, c w p 66$ and $c w p 84$, which are closely located within the genome, and are each represented on the microarray by two separate reporters. (c) Each line represents one gene within the CDO226-CDO272 genome region, encoding primarily flagellar proteins.

translation have been reported to suppress the production of ppGpp (Lund \& Kjeldgaard, 1972; Muto et al., 1975), the mediator of the stringent response. Transcripts of a putative ppGpp synthesis/degradation protein were also seen to be significantly upregulated in $C$. difficile after growth in the presence of both amoxicillin and clindamycin, and could possibly be responsible for the degradation of ppGpp. ppGpp would cause repression of ribosomal protein expression, and it is possible that this derepression is responsible for the observed increase in ribosomal protein transcripts.

The primary effect of growth in the presence of antibiotics appears to be on the surface composition of $C$. difficile. The region of the genome from $C D 2767-C D 2801$ encodes many surface-associated proteins, including cell wall biosynthesis and modification proteins (Calabi et al., 2001; Sebaihia et al., 2006). Also present are 13 genes encoding cell wall proteins (CWPs) containing the putative bacterial cell wallbinding Pfam domain PF04122; these are all paralogues of the slpA gene (Calabi et al., 2001; Karjalainen et al., 2001). In total, 27 of the 35 genes in this region (34 of 47 reporters) are over 1.3-fold upregulated by amoxicillin -6 significantly so - and the cluster as a whole is on average 2.3-fold upregulated. In addition to the $13 \mathrm{CWP}$-encoding genes present in this cluster, there are a further 16 localized throughout the genome. Each of these slpA paralogues is represented on the microarray by two separate reporters each printed in duplicate. On average, all microarray reporters representing CWP-encoding genes are upregulated in the presence of ampicillin (2.3-fold), clindamycin (1.5-fold) and metronidazole (1.3-fold). At least one reporter for $c w p 2, c w p 84, c w p 6$ and $c w p 7$ is significantly upregulated by amoxicillin (Fig. 4b) and for $c w p 21$ is upregulated by clindamycin, whereas $c w p 23$ is downregulated by clindamycin (data not shown). Antibiotic upregulation of $\operatorname{slp} A, c w p 66$ and $c w p 84$ transcription has been previously demonstrated in $C$. difficile, although the level of regulation varied between strains and antibiotics (Anne Collignon, personal communication).

Expression of genes encoding several cell wall enzymes was affected by treatment with amoxicillin, which mimics Dalanyl-D-alanine and disrupts cell wall synthesis (reviewed by Macheboeuf et al., 2006). $d l t A$ and $d a c F$ were upregulated, as was a gene cluster incorporating $m u r D, m r a Y$ and $m u r F$. All of the mur genes, which are involved in the peptidoglycan synthesis pathway, were upregulated at least 1.2-fold (mean value 1.9-fold). Two further genes were significantly induced by amoxicillin, encoding a penicillin-binding protein and a putative $\beta$-lactamase. In addition, induction was observed of a gene cluster encoding a penicillin-binding protein and a penicillin-binding protein repressor. Overall, all annotated penicillin-binding protein genes are either upregulated or downregulated by a mean of 1.7 -fold; none remain unaffected by amoxicillin. These results suggest that $C$. difficile downregulates the targets with highest affinity for amoxicillin, and upregulates all alternative pathways. A cell wall stimulon has recently been described for Staphylococcus aureus (Utaida et al., 2003), which may be upregulated in vancomycin intermediate-resistance strains (McAleese et al., 2006). Comparison between this regulon and those genes regulated in this study reveals some overlap; however, some genes have disparate regulation and many $S$. aureus genes are not identified in C. difficile.

Three principle components of the secretion apparatus, $\sec E$, $\sec Y$ and $\sec A 2$, were also upregulated by antibiotics, 
particularly amoxicillin (data not shown). Taken together with the upregulation of cell wall biosynthetic component transcripts, it is tempting to speculate that perhaps $C$. difficile alters the composition of its cell wall in response to antibiotics, perhaps producing a thicker or denser cell wall as a physical barrier to antibiotic penetration, similar to the mechanism that confers intermediate vancomycin resistance in S. aureus (reviewed by Appelbaum \& Bozdogan, 2004). However, this phenomenon has not been previously reported for $C$. difficile in response to antibiotics.

The region of the genome $C D 0226-C D 0272$ contains primarily genes encoding flagellar proteins. These genes appear to be co-ordinately upregulated after growth in the presence of amoxicillin and clindamycin by at least 1.6-fold in each case (mean values 3.0- and 2.3-fold, respectively), of which 12 (out of 40) are significantly upregulated by amoxicillin (Fig. 4c). The 11 other chemotaxis-related protein-encoding genes, present in two clusters within the genome, are also upregulated by amoxicillin by a mean of 1.4-fold, and by clindamycin by a mean of 1.6-fold (although only 2 genes are significantly regulated). The induction of flagellar protein-encoding transcripts and other chemotaxis-related proteins by both amoxicillin and clindamycin implies that cellular motility is increased in the presence of these antibiotics. The gut mucus of animals and humans serves as a chemoattractant for C. difficile, and chemotactic motility is positively correlated with the relative virulence of several $C$. difficile strains in a hamster model (Borriello et al., 1988). Increased motility could perhaps allow the bacterium to move down a concentration gradient of antibiotic, or to move towards higher nutrient concentrations.

\section{Concluding remarks}

Our data show that some components of the stress responses of $C$. difficile are similar to those observed in other organisms, whilst other components appear to be unique. The transcriptional profiles of $C$. difficile 630 are similar after growth in the presence of amoxicillin and clindamycin. In contrast, metronidazole treatment resulted in a very small change in transcription patterns. The effect of these antibiotics on the synthesis of CWPs merits further study. Further analysis of other genes identified in this study will enable targeted investigation into disruption of the genes, proteins and pathways shown to be necessary for stress survival, and therefore adaptation to the host environment and colonization.

\section{ACKNOWLEDGEMENTS}

We acknowledge B $\mu \mathrm{G} @$ S (the Bacterial Microarray Group at St George's, University of London, UK) for advice and supply of the microarray, and the Wellcome Trust for funding the multicollaborative microbial pathogen microarray facility under its Functional Genomics Resources Initiative. J.E. E. was supported by a studentship from the Wellcome Trust.

\section{REFERENCES}

Appelbaum, P. C. \& Bozdogan, B. (2004). Vancomycin resistance in Staphylococcus aureus. Clin Lab Med 24, 381-402.

Bartlett, J. G. (2006). Narrative review: the new epidemic of Clostridium difficile-associated enteric disease. Ann Intern Med 145, 758-764.

Boorstein, W. R., Ziegelhoffer, T. \& Craig, E. A. (1994). Molecular evolution of the HSP70 multigene family. J Mol Evol 38, 1-17.

Borriello, S. P., Welch, A. R., Barclay, F. E. \& Davies, H. A. (1988). Mucosal association by Clostridium difficile in the hamster gastrointestinal tract. J Med Microbiol 25, 191-196.

Calabi, E., Ward, S., Wren, B., Paxton, T., Panico, M., Morris, H., Dell, A., Dougan, G. \& Fairweather, N. (2001). Molecular characterization of the surface layer proteins from Clostridium difficile. Mol Microbiol 40, 1187-1199.

Freeman, J. \& Wilcox, M. H. (2001). Antibiotic activity against genotypically distinct and indistinguishable Clostridium difficile isolates. J Antimicrob Chemother 47, 244-246.

Gupta, R. S. (1995). Evolution of the chaperonin families (Hsp60, Hsp10 and Tcp-1) of proteins and the origin of eukaryotic cells. Mol Microbiol 15, 1-11.

Helmann, J. D., Wu, M. F., Kobel, P. A., Gamo, F. J., Wilson, M., Morshedi, M. M., Navre, M. \& Paddon, C. (2001). Global transcriptional response of Bacillus subtilis to heat shock. J Bacteriol 183, 7318-7328.

Hennequin, C., Porcheray, F., Waligora-Dupriet, A., Collignon, A., Barc, M., Bourlioux, P. \& Karjalainen, T. (2001). GroEL (Hsp60) of Clostridium difficile is involved in cell adherence. Microbiology 147, 87-96.

Hoper, D., Volker, U. \& Hecker, M. (2005). Comprehensive characterization of the contribution of individual SigB-dependent general stress genes to stress resistance of Bacillus subtilis. J Bacteriol 187, 2810-2826.

Hornbaek, T., Jakobsen, M., Dynesen, J. \& Nielsen, A. K. (2004). Global transcription profiles and intracellular $\mathrm{pH}$ regulation measured in Bacillus licheniformis upon external $\mathrm{pH}$ upshifts. Arch Microbiol 182, 467-474.

Jump, R. L., Pultz, M. J. \& Donskey, C. J. (2007). Vegetative Clostridium difficile survives in room air on moist surfaces and in gastric contents with reduced acidity: a potential mechanism to explain the association between proton pump inhibitors and $C$. difficile-associated diarrhea? Antimicrob Agents Chemother 51, 2883-2887.

Karjalainen, T., Waligora-Dupriet, A.-J., Cerquetti, M., Spigaglia, P., Maggioni, A., Mauri, P. \& Mastrantonio, P. (2001). Molecular and genomic analysis of genes encoding surface-anchored proteins from Clostridium difficile. Infect Immun 69, 3442-3446.

Lin, J. T., Connelly, M. B., Amolo, C., Otani, S. \& Yaver, D. S. (2005). Global transcriptional response of Bacillus subtilis to treatment with subinhibitory concentrations of antibiotics that inhibit protein synthesis. Antimicrob Agents Chemother 49, 1915-1926.

Loo, V. G., Poirier, L., Miller, M. A., Oughton, M., Libman, M. D., Michaud, S., Bourgault, A. M., Nguyen, T., Frenette, C. \& other authors (2005). A predominantly clonal multi-institutional outbreak of Clostridium difficile-associated diarrhea with high morbidity and mortality. N Engl J Med 353, 2442-2449.

Lund, E. \& Kjeldgaard, N. O. (1972). Metabolism of guanosine tetraphosphate in Escherichia coli. Eur J Biochem 28, 316-326.

Macheboeuf, P., Contreras-Martel, C., Job, V., Dideberg, O. \& Dessen, A. (2006). Penicillin binding proteins: key players in bacterial cell cycle and drug resistance processes. FEMS Microbiol Rev 30, 673-691.

McAleese, F., Wu, S. W., Sieradzki, K., Dunman, P., Murphy, E., Projan, S. \& Tomasz, A. (2006). Overexpression of genes of the cell 
wall stimulon in clinical isolates of Staphylococcus aureus exhibiting vancomycin-intermediate- $S$. aureus-type resistance to vancomycin. $J$ Bacteriol 188, 1120-1133.

McDonald, L. C., Killgore, G. E., Thompson, A., Owens, R. C., Jr, Kazakova, S. V., Sambol, S. P., Johnson, S. \& Gerding, D. N. (2005). An epidemic, toxin gene-variant strain of Clostridium difficile. $N$ Engl $J$ Med 353, 2433-2441.

Muto, A., Kimura, A. \& Osawa, S. (1975). Effects of some antibiotics on the stringent control of RNA synthesis in Escherichia coli. Mol Gen Genet 139, 321-327.

Pane-Farre, J., Jonas, B., Forstner, K., Engelmann, S. \& Hecker, M. (2006). The $\sigma^{\mathrm{B}}$ regulon in Staphylococcus aureus and its regulation. Int J Med Microbiol 296, 237-258.

Petersohn, A., Brigulla, M., Haas, S., Hoheisel, J. D., Volker, U. \& Hecker, M. (2001). Global analysis of the general stress response of Bacillus subtilis. J Bacteriol 183, 5617-5631.

Rider, J. E., Hacker, A., Mackintosh, C. A., Pegg, A. E., Woster, P. M. \& Casero, R. A., Jr (2007). Spermine and spermidine mediate protection against oxidative damage caused by hydrogen peroxide. Amino Acids 33, 231-240.

Rosen, R. \& Ron, E. Z. (2002). Proteome analysis in the study of the bacterial heat-shock response. Mass Spectrom Rev 21, 244-265.

Schumann, W. (2003). The Bacillus subtilis heat shock stimulon. Cell Stress Chaperones 8, 207-217.

Sebaihia, M., Wren, B. W., Mullany, P., Fairweather, N. F., Minton, N., Stabler, R., Thomson, N. R., Roberts, A. P., Cerdeno-Tarraga, A. M. \& other authors (2006). The multidrug-resistant human pathogen Clostridium difficile has a highly mobile, mosaic genome. Nat Genet 38, 779-786.

Shah, P., Marquart, M., Quin, L. R. \& Swiatlo, E. (2006). Cellular location of polyamine transport protein PotD in Streptococcus pneumoniae. FEMS Microbiol Lett 261, 235-237.
Stabler, R. A., Gerding, D. N., Songer, J. G., Drudy, D., Brazier, J. S., Trinh, H. T., Witney, A. A., Hinds, J. \& Wren, B. W. (2006). Comparative phylogenomics of Clostridium difficile reveals clade specificity and microevolution of hypervirulent strains. J Bacteriol 188, 7297-7305.

Tomas, C. A., Beamish, J. \& Papoutsakis, E. T. (2004). Transcriptional analysis of butanol stress and tolerance in Clostridium acetobutylicum. J Bacteriol 186, 2006-2018.

Utaida, S., Dunman, P. M., Macapagal, D., Murphy, E., Projan, S. J., Singh, V. K., Jayaswal, R. K. \& Wilkinson, B. J. (2003). Genome-wide transcriptional profiling of the response of Staphylococcus aureus to cell-wall-active antibiotics reveals a cell-wall-stress stimulon. Microbiology 149, 2719-2732.

van Schaik, W., van der Voort, M., Molenaar, D., Moezelaar, R., de Vos, W. M. \& Abee, T. (2007). Identification of the $\sigma^{\mathrm{B}}$ regulon of Bacillus cereus and conservation of $\sigma^{\mathrm{B}}$-regulated genes in low-GCcontent gram-positive bacteria. J Bacteriol 189, 4384-4390.

Ware, D., Jiang, Y., Lin, W. \& Swiatlo, E. (2006). Involvement of potD in Streptococcus pneumoniae polyamine transport and pathogenesis. Infect Immun 74, 352-361.

Warny, M., Pepin, J., Fang, A., Killgore, G., Thompson, A., Brazier, J., Frost, E. \& McDonald, L. C. (2005). Toxin production by an emerging strain of Clostridium difficile associated with outbreaks of severe disease in North America and Europe. Lancet 366, 1079-1084.

Wen, X. P., Pang, X. M., Matsuda, N., Kita, M., Inoue, H., Hao, Y. J., Honda, C. \& Moriguchi, T. (2007). Over-expression of the apple spermidine synthase gene in pear confers multiple abiotic stress tolerance by altering polyamine titers. Transgenic Res (in press).

Wiegert, T., Homuth, G., Versteeg, S. \& Schumann, W. (2001). Alkaline shock induces the Bacillus subtilis sigma(W) regulon. Mol Microbiol 41, 59-71.

Wust, J. \& Hardegger, U. (1983). Transferable resistance to clindamycin, erythromycin, and tetracycline in Clostridium difficile. Antimicrob Agents Chemother 23, 784-786. 